Article

\title{
Identification and Quantitative Characterization of PSORI-CM01, a Chinese Medicine Formula for Psoriasis Therapy, by Liquid Chromatography Coupled with an LTQ Orbitrap Mass Spectrometer
}

\author{
Shao-Dan Chen ${ }^{1,2,3}$, Chuan-Jian Lu ${ }^{1,2, *}$ and Rui-Zhi Zhao ${ }^{1,2}$ \\ 1 The Second Clinical College, Guangzhou University of Chinese Medicine, Guangzhou 510000, \\ China; E-Mails: shaodanchen@126.com (S.-D.C.); 13610241754@163.com (R.-Z.Z.) \\ 2 Guangdong Provincial Hospital of Chinese Medicine, Guangzhou 510000, China \\ 3 Postdoctoral Programme, Guangzhou University of Chinese Medicine, Guangzhou 510000, China \\ * Author to whom correspondence should be addressed; E-Mail: luchuanjian888@vip.sina.com; \\ Tel./Fax: +86-20-8188-7233.
}

Academic Editor: Derek J. McPhee

Received: 17 December 2014 / Accepted: 12 January 2015 / Published: 19 January 2015

\begin{abstract}
PSORI-CM01 is a Chinese medicine formula prepared from medicinal herbs and used in China for the treatment of psoriasis. However, the chemical constituents in PSORI-CM01 have not been clarified yet. In order to quickly define the chemical profiles and control the quality of PSORI-CM01 preparations, ultra-high liquid chromatography coupled with electrospray ionization hybrid linear trap quadrupole Orbitrap mass spectrometry (UHPLC-ESI-LTQ/Orbitrap-MS) was applied for simultaneous identification and quantification of multiple constituents. A total of 108 compounds, including organic acids, phenolic acids, flavonoids, and terpenoids, were identified or tentatively deduced on the base of their retention behaviors, MS and $\mathrm{MS}^{\mathrm{n}}$ data, or by comparing with reference substances and literature data. In addition, an optimized UHPLC-ESI-MS method was established for the quantitative determination of 14 marker compounds in different dosage forms of PSORI-CM01 preparations. The validation of the method, including spike recoveries, linearity, sensitivity (LOQ), precision, and repeatability, was carried out and demonstrated to be satisfied the requirements of quantitative analysis. This is the first report on the comprehensive determination of chemical constituents in PSORI-CM01 preparations by UHPLC-ESI-LTQ/Orbitrap mass spectrometry. The results suggested that the established methods would be a powerful and reliable analytical tool for the
\end{abstract}


characterization of multi-constituents in complex chemical system and quality control of TCM preparations.

Keywords: PSORI-CM01; UHPLC-ESI-LTQ/Orbitrap-MS; identification; quantification; TCM

\section{Introduction}

As known, the Chinese nation, with a history of more than 5000 years of civilization, largely relies on Traditional Chinese Medicine (TCM). TCM still plays a huge role in health care, disease prevention and treatment in China today. This is the reason why TCM has been deeply rooted in China and other Chinese cultural circles around the world for thousands of years. Traditional medicine, especially TCM, is not only an important and indispensable "alternative medicine" or "complementary medicine", but has also been entrusted with hopes for disease prevention and treatment in the future.

Traditional Chinese medicine prescriptions (TCMPs), or TCM formulae, which are applied according to certain compatibility rules, are the main and important clinical applications of TCM. However, TCMPs are facing difficulties because their effective constituents are unclear and sometimes inexplicable, which seriously restricts their development in the international market [1]. The chemical constituents of TCMPs are the key object of the study of TCM [2]. TCM is commonly considered to operate due to the synergistic effects of all the major and minor components in the medicines. Hence sensitive and comprehensive analytical techniques are needed to acquire a better understanding of the substance basis of TCM and to enhance the product quality control [3].

PSORI-CM01 was a novel formulated Chinese medicine used for psoriasis therapy [4]. It was optimized on the basis of a Chinese medicine formula Yin-Xie Ling, which was originated by Guo-Wei Xuan, the State Medical Master of China [5,6]. PSORI-CM01 was composed of seven herbs including Rhizoma Curcumae (E Zhu), Radix Paeoniae Rubra (Chi Shao), Sarcandra glabra (Zhong-Jie Feng), Radix Arnebiae (Zi Cao), Rhizoma Smilacis Glabrae (Tu-Fu Ling), Fructus Mume (Wu Mei), and Radix Glycyrrhizae (Gan Cao). Guo-Wei Xuan believed that one of the main causes of psoriasis is blood stasis. In this TCM formula PSORI-CM01, Curcumae bearing blood-activating and stasis-dissolving efficacy acts as the monarch drug, Paeoniae Rubra, Sarcandra glabra and Arnebiae, as the minister drugs, that together help Curcumae in activating blood and removing blood stasis. Smilacis Glabrae and Mume produce the effect of reducing the itch and together act as assistant drugs. Licorice, as a guide drug can mediate the other drugs' properties. When combined, the seven drugs have great therapeutic effects. Although the chemical constituents of the individual herbs have previously been well studied [7-15], little is known about the integrated chemical composition of PSORI-CM01. Unlike chemical drugs, botanical products contain a complex mixture of compounds. The contents of these compounds may be significantly affected by plant species, geographical sources, harvesting, processing and storage [16]. In addition, three dosage forms of PSORI-CM01 preparations (tablet, granules and decoction) are produced and used clinically in the Guangdong Provincial Hospital of Chinese Medicine. In order to guarantee drug safety and batch-to-batch consistency, quality control is therefore critically important for preparations such as PSORI-CM01. In recent years, the 
combination of Orbitrap technology with a linear ion trap, known as LTQ Orbitrap mass spectrometer were introduced, which could provide all the traditional MS and $\mathrm{MS}^{\mathrm{n}}$ scan functions using a linear IT and high mass accuracy measurements (errors within $5 \mathrm{ppm}$ ) [17]. Our previous study indicated that this analytical technique has the potential capability of simple, sensitive and reliable detection and identification of complex samples such as TCMs [9].

In the present study, a sensitive LC-ESI-MS ${ }^{\mathrm{n}}$ method was established for rapid separating, reliable identifying and quantifying the multiple components in PSORI-CM01 preparations, by using a hybrid LTQ-Orbitrap mass spectrometer coupled with an UHPLC system. The qualitative analysis was carried out in negative ionization mode to acquire accurate mass data in full scan mode and MS/MS in a data dependent product ion spectrum. Further, 14 reference compounds were quantitatively determined in negative ionization mode and eight samples of PSORI-CM01 preparations were analyzed for assessment of quality consistence.

\section{Results and Discussion}

\subsection{Optimization of Chromatographic Conditions}

To improve the resolution and sensitivity of the analysis but reduce the analytical time, the mobile phase system was optimized. To inhibit ionization of the acidic ingredients in PSORI-CM01, formic acid was added to the mobile phase. Two mobile phase systems, methanol-aqueous solution and acetonitrile-aqueous solution were compared. Both negative and positive modes were examined. Generally, in positive mode, low abundance of $[\mathrm{M}+\mathrm{H}]^{+},\left[\mathrm{M}+\mathrm{NH}_{4}\right]^{+}$ions and few product ions were observed, while, in negative ion mode, a series of $[\mathrm{M}-\mathrm{H}]^{-}$ions and/or adduct ions $\left([\mathrm{M}+\mathrm{HCOOH}-\mathrm{H}]^{-}\right)$ appeared with sufficient abundance. Thus the negative ion mode was chosen and the $[\mathrm{M}-\mathrm{H}]^{-} /\left([\mathrm{M}+\mathrm{HCOOH}-\mathrm{H}]^{-}\right)$ions were further subjected to $\mathrm{LC}-\mathrm{MS}^{\mathrm{n}}$ analysis. For the extracted target ions in full scan mode, an accurate mass limit of $5 \mathrm{ppm}$ accurate mass filter limit was used to characterize "real" compounds signals from the background peaks, as well as to increase the signal-to-noise ratio for each analyte.

\subsection{Identification of Chemical Constituents in PSORI-CM01 Preparations}

The reference substances and PSORI-CM01 sample were analyzed by using the optimized LC-ESI-MS ${ }^{\mathrm{n}}$ method. The TIC chromatogram of PSORI-CM01 sample in negative ESI mode was shown in Figure 1, and 108 peaks were observed in PSORI-CM01 sample. The MS data showed high precision with all the mass accuracy within $5 \mathrm{ppm}$. For most of the constituents, $[\mathrm{M}-\mathrm{H}]^{-}$ions were observed. Due to the use of formic acid in mobile phase, there were adduct ions of $[\mathrm{M}+46-\mathrm{H}]^{-}$corresponding to $[\mathrm{M}+\mathrm{HCOOH}-\mathrm{H}]^{-}$in negative ion mode. These results provided valuable information for confirming accurate molecular weight and composition of the constituents. 


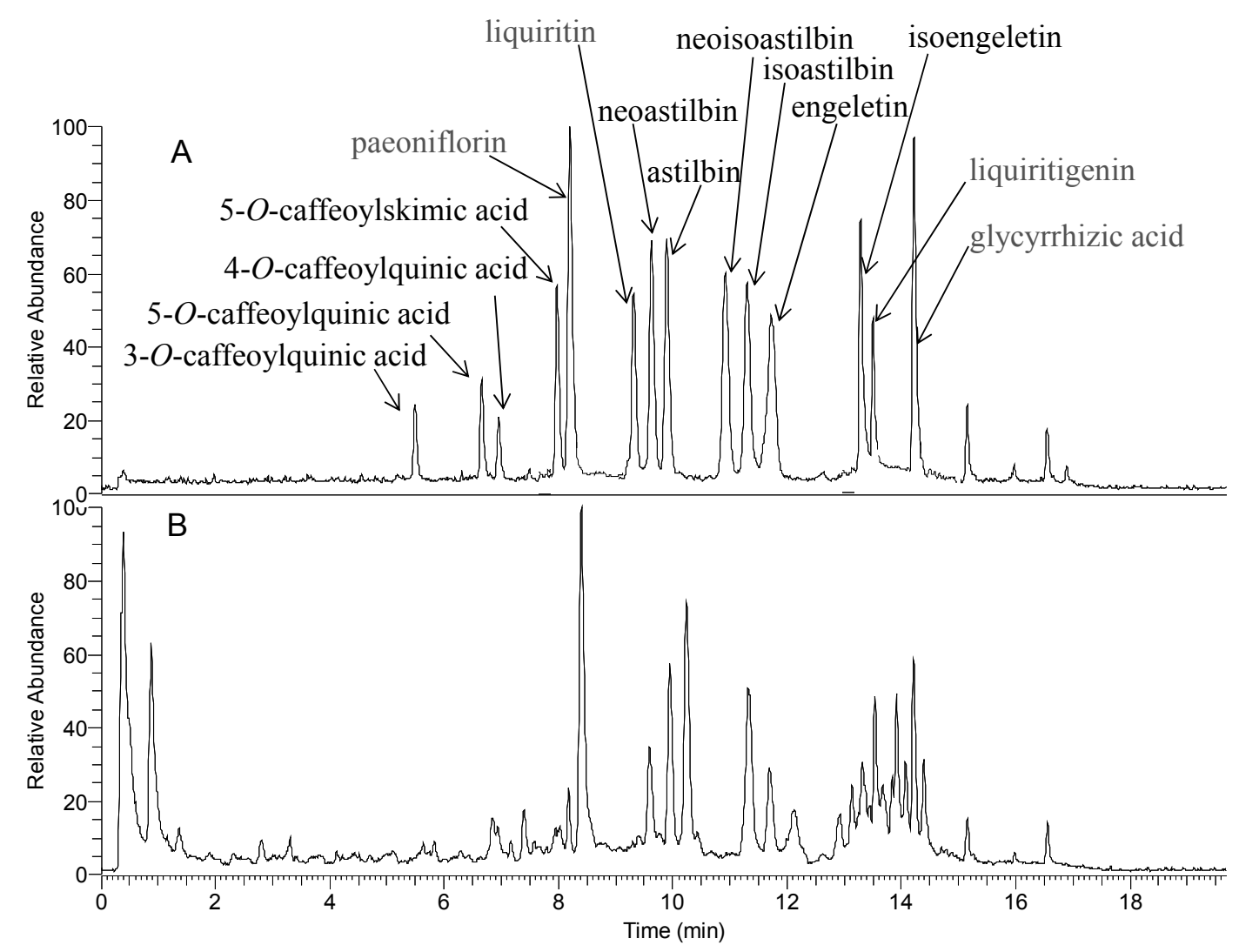

Figure 1. UHPLC-(-) ESI-MS total ion chromatograms of a mixture of fourteen standards (A) and PSORI-CM01 (B).

One hundred and eight compounds were tentatively identified on the basis of their retention behaviors, accurate molecular weights and $\mathrm{MS}^{\mathrm{n}}$ fragment data, or by comparison with reference substances or literature data. Corresponding quasimolecular ions and their fragment ions in the $\mathrm{MS}^{\mathrm{n}}$ spectra were listed in Table 1. The identified compounds can be classified into four classes including organic acids, phenolic acids, flavonoids, and terpenoids, which were mainly from Radix Paeoniae Rubra, Rhizoma Smilacis Glabrae, Sarcandra glabra, and Radix Glycyrrhizae. Monoterpene glucosides and phenolic acids are the main constituents of Paeoniae Rubra, caffeic acid derivatives and flavonoids are abundant in Sarcandra glabra, flavonoids and phenolic compounds are mainly from Rhizoma Smilacis Glabrae, while flavonoids and triterpene glucoside are from Radix Glycyrrhizae. To some extent, the UHPLC-ESI-MS chromatogram reflected the rationale of PSORI-CM01. However, the characteristic constituents (sesquiterpenoids and curcuminoids) from the monarch drug Rhizoma Curcumae were not detected.

Among the 108 compounds, 3-O-caffeoylquinic acid (16), 5-O-caffeoylquinic acid (22), 4-O-caffeoylquinic acid (24), 5-O-caffeoylskimic acid (39), paeoniflorin (40), liquiritin (51), neoastilbin (56), astilbin (60), neoisoastilbin (64), isoastilbin (67), engeletin (79), isoengeletin (82), liquiritigenin (87) and glycyrrhizic acid (107) were the main components in PSORI-CM01. Besides, 3-O-caffeoylquinic acid (16), paeoniflorin (40), liquiritin (51), astilbin (60), engeletin (79), liquiritigenin (87) and glycyrrhizic acid (107) have been reported to have multiple biological activities such as anti-inflammation, immunoregulation and anti-tumor properties [18-23], which are related to psoriasis. Thus, these 14 ingredients were selected as markers for quality control. 
Table 1. Identification of the chemical constituents of PSORI-CM01 by UHPLC-ESI-MS ${ }^{\mathrm{n}}$ analysis.

\begin{tabular}{|c|c|c|c|c|c|c|c|c|c|}
\hline $\begin{array}{l}\text { Peak } \\
\text { No. }\end{array}$ & $\underset{(\min )}{t \mathrm{R}}$ & $\begin{array}{l}\text { Selected } \\
\text { Ion }\end{array}$ & $\begin{array}{c}\text { Observed } \\
\text { Mass }(m / z)\end{array}$ & $\begin{array}{l}\text { Calculated } \\
\text { Mass }(m / z)\end{array}$ & Formula & $\begin{array}{l}\text { MS/MS Patterns } \\
\text { Fragmentation }\end{array}$ & Identifieation & Source $^{\text {a }}$ & Reference \\
\hline 1 & 0.41 & {$[\mathrm{M}-\mathrm{H}]$} & 191.0559 & 191.0556 & $\mathrm{C}_{7} \mathrm{H}_{12} \mathrm{O}_{6}$ & $191 \rightarrow 173,127,109$ & quinic acid & $\mathrm{Sa}$ & {$[8]$} \\
\hline 2 & 0.51 & {$[\mathrm{M}-\mathrm{H}]$} & 133.0140 & 133.0137 & $\mathrm{C}_{4} \mathrm{H}_{6} \mathrm{O}_{5}$ & $133 \rightarrow 115,87,71$ & malic acid & M & \\
\hline 3 & 0.60 & {$[\mathrm{M}-\mathrm{H}]$} & 173.0451 & 173.0450 & $\mathrm{C}_{7} \mathrm{H}_{10} \mathrm{O}_{5}$ & $173 \rightarrow 155,145,129$ & shikimic acid & $\mathrm{Sm}$ & [9] \\
\hline 4 & 0.88 & {$[\mathrm{M}-\mathrm{H}]$} & 191.0198 & 191.0192 & $\mathrm{C}_{6} \mathrm{H}_{8} \mathrm{O}_{7}$ & $191 \rightarrow 173,155 \rightarrow 111$ & citric acid & M & \\
\hline 5 & 0.98 & {$[\mathrm{M}-\mathrm{H}]$} & 115.0037 & 115.0031 & $\mathrm{C}_{4} \mathrm{H}_{4} \mathrm{O}_{4}$ & $115 \rightarrow 97,71$ & fumaric acid & $\mathrm{Sa}$ & {$[8]$} \\
\hline 6 & 2.16 & {$[\mathrm{M}-\mathrm{H}]$} & 169.0142 & 169.0137 & $\mathrm{C}_{7} \mathrm{H}_{6} \mathrm{O}_{5}$ & $169 \rightarrow 125$ & gallic acid & $\mathrm{P}$ & {$[24,25]$} \\
\hline 7 & 2.60 & {$[\mathrm{M}-\mathrm{H}]$} & 331.0663 & 331.0665 & $\mathrm{C}_{13} \mathrm{H}_{16} \mathrm{O}_{10}$ & $331 \rightarrow 169$ & gallic acid-1-O-glucoside & $\mathrm{P}$ & \\
\hline 8 & 3.28 & {$[\mathrm{M}-\mathrm{H}]$} & 315.0716 & 315.0716 & $\mathrm{C}_{13} \mathrm{H}_{16} \mathrm{O}_{9}$ & $315 \rightarrow 297,247,153$ & protocatechuic acid-4- $O$-glucoside & $\mathrm{P}, \mathrm{C}$ & \\
\hline 9 & 3.64 & {$[\mathrm{M}-\mathrm{H}]$} & 197.0453 & 197.0450 & $\mathrm{C}_{9} \mathrm{H}_{10} \mathrm{O}_{5}$ & $197 \rightarrow 153,123$ & syringic acid & $\mathrm{Sa}, \mathrm{Sm}$ & [9] \\
\hline 10 & 3.69 & {$[\mathrm{M}-\mathrm{H}]$} & 315.0716 & 315.0716 & $\mathrm{C}_{13} \mathrm{H}_{16} \mathrm{O}_{9}$ & $315 \rightarrow 169$ & gallic acid-1-O-rhamnoside & $\mathrm{P}$ & \\
\hline 11 & 3.78 & {$[\mathrm{M}-\mathrm{H}]$} & 153.0192 & 153.0188 & $\mathrm{C}_{7} \mathrm{H}_{6} \mathrm{O}_{4}$ & $153 \rightarrow 109$ & protocatechuic acid & $\mathrm{Sa}, \mathrm{Sm}, \mathrm{C}$ & {$[8,9]$} \\
\hline 12 & 3.82 & {$[\mathrm{M}-\mathrm{H}]$} & 493.1208 & 493.1193 & $\mathrm{C}_{19} \mathrm{H}_{26} \mathrm{O}_{15}$ & $493 \rightarrow 313,169$ & gallic acid-1-O-glucosyl- $(6 \rightarrow 1)$-glucoside & $\mathrm{P}$ & \\
\hline 13 & 4.02 & {$[\mathrm{M}-\mathrm{H}]$} & 359.0977 & 359.0978 & $\mathrm{C}_{15} \mathrm{H}_{20} \mathrm{O}_{10}$ & $359 \rightarrow 197,179$ & syringic acid-4-O-glucoside & $\mathrm{Sa}, \mathrm{Sm}$ & [9] \\
\hline 14 & 4.83 & {$[\mathrm{M}-\mathrm{H}]$} & 181.0495 & 181.0450 & $\mathrm{C}_{9} \mathrm{H}_{10} \mathrm{O}_{5}$ & $197 \rightarrow 169,133$ & 3,5-dimethoxy-4-hydroxy-benzaldehyde & $\mathrm{Sa}$ & \\
\hline 15 & 4.93 & {$[\mathrm{M}-\mathrm{H}]$} & 137.0243 & 137.0239 & $\mathrm{C}_{7} \mathrm{H}_{6} \mathrm{O}_{3}$ & $137 \rightarrow 109,93$ & 4-hydroxybenzoic acid & $\begin{array}{l}\mathrm{C}, \mathrm{M}, \mathrm{Sm}, \\
\quad \mathrm{Sa}\end{array}$ & \\
\hline 16 & 5.01 & {$[\mathrm{M}-\mathrm{H}]$} & 353.0873 & 353.0873 & $\mathrm{C}_{16} \mathrm{H}_{18} \mathrm{O}_{9}$ & $353 \rightarrow 191,179 \rightarrow 135$ & 3-O-caffeoylquinic acid & $\mathrm{Sa}$ & {$[8]$} \\
\hline 17 & 5.24 & {$[\mathrm{M}-\mathrm{H}]$} & 165.0556 & 165.0552 & $\mathrm{C}_{9} \mathrm{H}_{10} \mathrm{O}_{3}$ & $165 \rightarrow 151,135$ & paeonal & $\mathrm{P}$ & {$[24,25]$} \\
\hline 18 & 5.31 & {$[\mathrm{M}-\mathrm{H}]$} & 345.1182 & 345.1186 & $\mathrm{C}_{15} \mathrm{H}_{22} \mathrm{O}_{9}$ & $345 \rightarrow 183$ & $\begin{array}{l}\text { (3,5-dimethoxy-4-hydroxyphenyl)methy } \\
\text {-O-glucoside }\end{array}$ & $\mathrm{Sa}$ & \\
\hline 19 & 5.59 & {$[\mathrm{M}-\mathrm{H}]$} & 183.0296 & 183.0293 & $\mathrm{C}_{8} \mathrm{H}_{8} \mathrm{O}_{5}$ & $183 \rightarrow 124$ & methyl gallate & $\mathrm{P}$ & \\
\hline 20 & 6.20 & {$[\mathrm{M}-\mathrm{H}]$} & 609.1445 & 609.1456 & $\mathrm{C}_{27} \mathrm{H}_{30} \mathrm{O}_{16}$ & $609 \rightarrow 445,301$ & rutin & M & \\
\hline 21 & 6.20 & {$[\mathrm{M}-\mathrm{H}]$} & 495.1498 & 495.1503 & $\mathrm{C}_{23} \mathrm{H}_{28} \mathrm{O}_{12}$ & $495 \rightarrow 465,333,281$ & oxypaeoniflorin & $\mathrm{P}$ & \\
\hline
\end{tabular}


Table 1. Cont.

\begin{tabular}{|c|c|c|c|c|c|c|c|c|c|}
\hline $\begin{array}{l}\text { Peak } \\
\text { No. }\end{array}$ & $\begin{array}{c}t \mathrm{R} \\
(\mathrm{min})\end{array}$ & $\begin{array}{c}\text { Selected } \\
\text { Ion }\end{array}$ & $\begin{array}{c}\text { Observed } \\
\operatorname{Mass}(m / z)\end{array}$ & $\begin{array}{l}\text { Calculated } \\
\operatorname{Mass}(m / z)\end{array}$ & Formula & $\begin{array}{c}\text { MS/MS Patterns } \\
\text { Fragmentation }\end{array}$ & Identifieation & Source $^{a}$ & Reference \\
\hline 22 & 6.12 & {$[\mathrm{M}-\mathrm{H}]$} & 353.0872 & 353.0873 & $\mathrm{C}_{16} \mathrm{H}_{18} \mathrm{O}_{9}$ & $353 \rightarrow 191,179,135$ & 5-O-caffeoylquinic acid & $\mathrm{Sa}$ & [8] \\
\hline 23 & 6.17 & {$[\mathrm{M}-\mathrm{H}]$} & 289.0713 & 289.0712 & $\mathrm{C}_{15} \mathrm{H}_{14} \mathrm{O}_{6}$ & $289 \rightarrow 245,205,179$ & $(+)$-catechin & $\mathrm{P}, \mathrm{Sm}$ & {$[9]$} \\
\hline 24 & 6.18 & {$[\mathrm{M}-\mathrm{H}]$} & 335.0767 & 335.0767 & $\mathrm{C}_{16} \mathrm{H}_{16} \mathrm{O}_{8}$ & $335 \rightarrow 289,179,135,111$ & 3-O-caffeoylshikimic acid & $\mathrm{Sa}, \mathrm{Sm}$ & {$[9]$} \\
\hline 25 & 6.21 & {$[\mathrm{M}-\mathrm{H}]$} & 321.0246 & 321.0247 & $\mathrm{C}_{14} \mathrm{H}_{10} \mathrm{O}_{9}$ & $321 \rightarrow 169$ & $p$-digallic acid & $\mathrm{P}$ & \\
\hline 26 & 6.41 & {$[\mathrm{M}-\mathrm{H}]$} & 353.0873 & 353.0873 & $\mathrm{C}_{16} \mathrm{H}_{18} \mathrm{O}_{9}$ & $353 \rightarrow 191,179,135$ & 4-O-caffeoylquinic acid & $\mathrm{Sa}$ & {$[8]$} \\
\hline 27 & 6.43 & {$[\mathrm{M}-\mathrm{H}]$} & 469.1129 & 469.1135 & $\mathrm{C}_{24} \mathrm{H}_{22} \mathrm{O}_{10}$ & $469 \rightarrow 423,371,315,289$ & $\begin{array}{l}\text { 8-[ } \beta \text {-(3,4-dihydroxyphenyl)- } \alpha \text {-carboxyl } \\
\text {-3-xoxpropyl]-substituted catechin }\end{array}$ & $\mathrm{Sm}$ & [9] \\
\hline 28 & 6.45 & {$[\mathrm{M}-\mathrm{H}]$} & 179.0345 & 179.0344 & $\mathrm{C}_{9} \mathrm{H}_{8} \mathrm{O}_{4}$ & $179 \rightarrow 135,85$ & caffeic acid & $\mathrm{Sa}, \mathrm{Sm}, \mathrm{A}$ & {$[8,9]$} \\
\hline 29 & 6.59 & {$[\mathrm{M}+\mathrm{COOH}]$} & 447.1500 & 447.1503 & $\mathrm{C}_{18} \mathrm{H}_{26} \mathrm{O}_{10}$ & $447 \rightarrow 401,349,317,191$ & phenylmethyl-glucoside- $(6 \rightarrow 1)$-apiose & $\mathrm{M}$ & \\
\hline 30 & 6.62 & {$[\mathrm{M}-\mathrm{H}]$} & 221.0452 & 221.0450 & $\mathrm{C}_{11} \mathrm{H}_{10} \mathrm{O}_{5}$ & $221 \rightarrow 206$ & fraxidin & $\mathrm{Sa}$ & {$[8]$} \\
\hline 31 & 6.67 & {$[\mathrm{M}-\mathrm{H}]$} & 369.0821 & 369.0822 & $\mathrm{C}_{16} \mathrm{H}_{18} \mathrm{O}_{10}$ & $369 \rightarrow 207$ & fraxin & $\mathrm{Sa}$ & {$[8]$} \\
\hline 32 & 6.87 & {$[\mathrm{M}-\mathrm{H}]$} & 435.1289 & 435.1291 & $\mathrm{C}_{21} \mathrm{H}_{24} \mathrm{O}_{10}$ & $435 \rightarrow 273$ & isoliquiritigenin-7-O-glucoside & $\mathrm{Sa}, \mathrm{P}$ & {$[8]$} \\
\hline 33 & 6.87 & {$[\mathrm{M}-\mathrm{H}]$} & 431.1914 & 431.1917 & $\mathrm{C}_{20} \mathrm{H}_{32} \mathrm{O}_{10}$ & $431 \rightarrow 385,223,205,153$ & drovomifoliol- $O$-glucopyranoside & $\mathrm{Sa}$ & {$[8]$} \\
\hline 34 & 6.96 & {$[\mathrm{M}-\mathrm{H}]$} & 433.2070 & 433.2074 & $\mathrm{C}_{20} \mathrm{H}_{34} \mathrm{O}_{10}$ & $433 \rightarrow 387,369,225$ & dihydrovomifoliol- $O$-glucoside & $\mathrm{Sa}$ & {$[8]$} \\
\hline 35 & 7.13 & {$[\mathrm{M}-\mathrm{H}]$} & 335.0767 & 335.0767 & $\mathrm{C}_{16} \mathrm{H}_{16} \mathrm{O}_{8}$ & $335 \rightarrow 289,179,135,111$ & 4- $O$-caffeoylshikimic acid & $\mathrm{Sa}, \mathrm{Sm}$ & {$[9]$} \\
\hline 36 & 7.20 & {$[\mathrm{M}-\mathrm{H}]$} & 289.0712 & 289.0712 & $\mathrm{C}_{15} \mathrm{H}_{14} \mathrm{O}_{6}$ & $289 \rightarrow 245,205,179$ & epi-catechin & $\mathrm{P}, \mathrm{Sm}$ & {$[9]$} \\
\hline 37 & 7.23 & {$[\mathrm{M}+\mathrm{COOH}]$} & 525.1604 & 481.1710 & $\mathrm{C}_{23} \mathrm{H}_{30} \mathrm{O}_{11}$ & $525 \rightarrow 479,465,121$ & albiflorin & $\mathrm{P}$ & {$[24]$} \\
\hline 38 & 7.28 & {$[\mathrm{M}-\mathrm{H}]$} & 207.0296 & 207.0293 & $\mathrm{C}_{10} \mathrm{H}_{8} \mathrm{O}_{5}$ & $207 \rightarrow 192$ & fraxetin & $\mathrm{Sa}$ & {$[8]$} \\
\hline 39 & 7.38 & {$[\mathrm{M}-\mathrm{H}]$} & 335.0767 & 335.0767 & $\mathrm{C}_{16} \mathrm{H}_{16} \mathrm{O}_{8}$ & $335 \rightarrow 289,179,135,111$ & 5-O-caffeoylshikimic acid & $\mathrm{Sa}, \mathrm{Sm}$ & {$[9]$} \\
\hline 40 & 7.61 & {$[\mathrm{M}+\mathrm{COOH}]$} & 525.1604 & 481.1710 & $\mathrm{C}_{23} \mathrm{H}_{30} \mathrm{O}_{11}$ & $525 \rightarrow 479,327,283,161$ & paeoniflorin & $\mathrm{P}$ & {$[24,25]$} \\
\hline 41 & 7.72 & {$[\mathrm{M}-\mathrm{H}]$} & 471.1863 & 471.1866 & $\mathrm{C}_{22} \mathrm{H}_{32} \mathrm{O}_{11}$ & $471 \rightarrow 425,263$ & sarcaglaboside G & $\mathrm{Sa}$ & {$[8]$} \\
\hline 42 & 7.81 & {$[\mathrm{M}-\mathrm{H}]$} & 339.0715 & 339.0716 & $\mathrm{C}_{15} \mathrm{H}_{16} \mathrm{O}_{9}$ & $339 \rightarrow 193,165,137$ & 6,7,8-trihydroxycoumarin-7-rhamnoside & $\mathrm{Sa}, \mathrm{Sm}$ & {$[9]$} \\
\hline 43 & 7.94 & {$[\mathrm{M}-\mathrm{H}]$} & 629.1514 & 629.1506 & $\mathrm{C}_{30} \mathrm{H}_{30} \mathrm{O}_{15}$ & $\begin{array}{c}629 \rightarrow 483,475,449,303 \\
285\end{array}$ & $\begin{array}{l}\text { 8-[}[\beta \text {-(3,4-dihydroxyphenyl)- } \alpha \text {-carboxyl-3- } \\
\text { oxopropyl]-substituted neoastilbin }\end{array}$ & $\mathrm{Sm}$ & [9] \\
\hline
\end{tabular}


Table 1. Cont.

\begin{tabular}{|c|c|c|c|c|c|c|c|c|c|}
\hline $\begin{array}{l}\text { Peak } \\
\text { No. }\end{array}$ & $\begin{array}{c}t \mathbf{R} \\
(\mathrm{min})\end{array}$ & $\begin{array}{c}\text { Selected } \\
\text { Ion }\end{array}$ & $\begin{array}{c}\text { Observed } \\
\operatorname{Mass}(m / z)\end{array}$ & $\begin{array}{l}\text { Calculated } \\
\operatorname{Mass}(m / z)\end{array}$ & Formula & $\begin{array}{l}\text { MS/MS Patterns } \\
\text { Fragmentation }\end{array}$ & Identifieation & Source $^{a}$ & Reference \\
\hline 44 & 8.05 & {$[\mathrm{M}-\mathrm{H}]$} & 473.2022 & 473.2023 & $\mathrm{C}_{22} \mathrm{H}_{34} \mathrm{O}_{11}$ & 473.202 & sarcaglaboside $\mathrm{H}$ & $\mathrm{Sa}$ & {$[8]$} \\
\hline 45 & 8.16 & {$[\mathrm{M}-\mathrm{H}]$} & 629.1514 & 629.1506 & $\mathrm{C}_{30} \mathrm{H}_{30} \mathrm{O}_{15}$ & $\begin{array}{c}629 \rightarrow 483,475,449,303, \\
285\end{array}$ & $\begin{array}{l}\text { 8-[ } \beta \text {-(3,4-dihydroxyphenyl)- } \alpha \text {-carboxyl-3- } \\
\text { oxopropyl]-substituted astilbin }\end{array}$ & $\mathrm{Sm}$ & [9] \\
\hline 46 & 8.18 & {$[\mathrm{M}-\mathrm{H}]$} & 565.1552 & 565.1557 & $\mathrm{C}_{26} \mathrm{H}_{30} \mathrm{O}_{14}$ & $565 \rightarrow 313,193$ & $\begin{array}{c}(2 R / 2 S) \text {-naringenin-6- } C \text { - } \beta \text {-D-glucopy ranoside- } \\
(6-1) \text {-apiose }\end{array}$ & $\mathrm{Sa}$ & {$[8]$} \\
\hline 47 & 8.27 & {$[\mathrm{M}-\mathrm{H}]$} & 537.1025 & 537.1033 & $\mathrm{C}_{27} \mathrm{H}_{22} \mathrm{O}_{12}$ & $537 \rightarrow 493,295,159,109$ & lithospermic acid & A & \\
\hline 48 & 8.33 & {$[\mathrm{M}-\mathrm{H}]$} & 565.1550 & 565.1557 & $\mathrm{C}_{26} \mathrm{H}_{30} \mathrm{O}_{14}$ & $565 \rightarrow 313,193$ & $\begin{array}{c}(2 R / 2 S) \text {-naringenin-6-C-glucopyranoside-(6- } \\
1 \text { )-apiose }\end{array}$ & $\mathrm{Sa}$ & [9] \\
\hline 49 & 8.52 & {$[\mathrm{M}-\mathrm{H}]$} & 629.1514 & 629.1506 & $\mathrm{C}_{30} \mathrm{H}_{30} \mathrm{O}_{15}$ & $\begin{array}{c}629 \rightarrow 483,475,449,303, \\
285\end{array}$ & $\begin{array}{l}\text { 8-[ } \beta \text {-(3,4-dihydroxyphenyl)- } \alpha \text {-carboxyl-3- } \\
\text { oxopropyl]-substituted }\end{array}$ & $\mathrm{Sm}$ & [9] \\
\hline 50 & 8.57 & {$[\mathrm{M}-\mathrm{H}]$} & 221.0452 & 221.0450 & $\mathrm{C}_{11} \mathrm{H}_{10} \mathrm{O}_{5}$ & $221 \rightarrow 206,191,163$ & isofraxidin & $\mathrm{Sa}$ & [8] \\
\hline 51 & 8.62 & {$[\mathrm{M}-\mathrm{H}]$} & 417.1186 & 417.1186 & $\mathrm{C}_{21} \mathrm{H}_{22} \mathrm{O}_{9}$ & $417 \rightarrow 255$ & liquiritin & G & {$[26]$} \\
\hline 52 & 8.62 & {$[\mathrm{M}-\mathrm{H}]$} & 549.1607 & 549.1608 & $\mathrm{C}_{26} \mathrm{H}_{30} \mathrm{O}_{13}$ & $549 \rightarrow 429,255$ & liquiritin apioside & $\mathrm{G}$ & {$[26]$} \\
\hline 53 & 8.77 & {$[\mathrm{M}-\mathrm{H}]$} & 451.1025 & 451.1029 & $\mathrm{C}_{24} \mathrm{H}_{20} \mathrm{O}_{9}$ & $451 \rightarrow 341,299$ & cinchonain Ia & $\mathrm{Sm}$ & \\
\hline 54 & 8.80 & {$[\mathrm{M}-\mathrm{H}]$} & 303.0505 & 303.0505 & $\mathrm{C}_{15} \mathrm{H}_{12} \mathrm{O}_{7}$ & $303 \rightarrow 285$ & taxifolin & $\mathrm{Sm}$ & [9] \\
\hline 55 & 8.82 & {$[\mathrm{M}-\mathrm{H}]$} & 300.9986 & 300.9984 & $\mathrm{C}_{14} \mathrm{H}_{6} \mathrm{O}_{8}$ & $301 \rightarrow 257$ & gallogen & $\mathrm{P}$ & \\
\hline 56 & 8.84 & {$[\mathrm{M}-\mathrm{H}]$} & 449.1088 & 449.1084 & $\mathrm{C}_{21} \mathrm{H}_{22} \mathrm{O}_{11}$ & $449 \rightarrow 303,285$ & neoastilbin & $\mathrm{Sa}, \mathrm{Sm}$ & {$[8,9]$} \\
\hline 57 & 8.98 & {$[\mathrm{M}-\mathrm{H}]$} & 629.1514 & 629.1506 & $\mathrm{C}_{30} \mathrm{H}_{30} \mathrm{O}_{15}$ & $\begin{array}{c}629 \rightarrow 483,475,449,303, \\
285\end{array}$ & $\begin{array}{l}\text { 8-[ } \beta \text {-(3,4-dihydroxyphenyl)- } \alpha \text {-carboxyl-3- } \\
\text { oxopropyl]-substituted isoastilbin }\end{array}$ & $\mathrm{Sm}$ & [9] \\
\hline 58 & 8.96 & {$[\mathrm{M}-\mathrm{H}]$} & 631.1656 & 631.1663 & $\mathrm{C}_{30} \mathrm{H}_{32} \mathrm{O}_{15}$ & $631 \rightarrow 613,491,399,169$ & galloyl paeoniflorin & $\mathrm{P}$ & \\
\hline 59 & 9.02 & {$[\mathrm{M}-\mathrm{H}]$} & 477.0668 & 477.0669 & $\mathrm{C}_{21} \mathrm{H}_{18} \mathrm{O}_{13}$ & $477 \rightarrow 301$ & quercetin-3-O-glucruronide & $\mathrm{Sa}$ & {$[8]$} \\
\hline 60 & 9.02 & {$[\mathrm{M}-\mathrm{H}]$} & 449.1088 & 449.1084 & $\mathrm{C}_{21} \mathrm{H}_{22} \mathrm{O}_{11}$ & $449 \rightarrow 303,285$ & astilbin & $\mathrm{Sa}, \mathrm{Sm}$ & {$[8,9]$} \\
\hline 61 & 9.05 & {$[\mathrm{M}-\mathrm{H}]$} & 521.1295 & 521.1295 & $\mathrm{C}_{24} \mathrm{H}_{26} \mathrm{O}_{13}$ & $521 \rightarrow 359,197$ & rosmarinic acid-4-O-glucoside & $\mathrm{Sa}$ & \\
\hline 62 & 9.38 & {$[\mathrm{M}-\mathrm{H}]$} & 939.1089 & 939.1104 & $\mathrm{C}_{41} \mathrm{H}_{32} \mathrm{O}_{26}$ & $939 \rightarrow 769,617,393,317$ & penta- $O$-galloyl-glucose & $\mathrm{P}$ & \\
\hline
\end{tabular}


Table 1. Cont.

\begin{tabular}{|c|c|c|c|c|c|c|c|c|c|}
\hline $\begin{array}{c}\text { Peak } \\
\text { No. }\end{array}$ & $\begin{array}{c}t \mathrm{R} \\
(\mathrm{min}) \\
\end{array}$ & $\begin{array}{c}\text { Selected } \\
\text { Ion } \\
\end{array}$ & $\begin{array}{c}\text { Observed } \\
\text { Mass }(m / z)\end{array}$ & $\begin{array}{l}\text { Calculated } \\
\text { Mass }(m / z)\end{array}$ & Formula & $\begin{array}{c}\text { MS/MS Patterns } \\
\text { Fragmentation }\end{array}$ & Identifieation & Source $^{a}$ & Reference \\
\hline 63 & 9.49 & {$[\mathrm{M}-\mathrm{H}]$} & 515.1184 & 515.1190 & $\mathrm{C}_{25} \mathrm{H}_{24} \mathrm{O}_{12}$ & $515 \rightarrow 353$ & dicaffeoylquinic acid & $\mathrm{Sa}$ & \\
\hline 64 & 9.64 & {$[\mathrm{M}-\mathrm{H}]$} & 449.1088 & 449.1084 & $\mathrm{C}_{21} \mathrm{H}_{22} \mathrm{O}_{11}$ & $449 \rightarrow 303,285$ & neoisoastilbin & $\mathrm{Sa}, \mathrm{Sm}$ & {$[8,9]$} \\
\hline 65 & 9.68 & {$[\mathrm{M}-\mathrm{H}]$} & 717.1442 & 717.1456 & $\mathrm{C}_{36} \mathrm{H}_{30} \mathrm{O}_{16}$ & $717 \rightarrow 519,475,321$ & caffeic acid tetramer & $\mathrm{A}$ & \\
\hline 66 & 9.78 & {$[\mathrm{M}-\mathrm{H}]$} & 187.0974 & 187.0970 & $\mathrm{C}_{9} \mathrm{H}_{16} \mathrm{O}_{4}$ & $187 \rightarrow 142,125$ & nonandioic acid & $\mathrm{P}, \mathrm{G}$ & \\
\hline 67 & 9.84 & {$[\mathrm{M}-\mathrm{H}]$} & 449.1088 & 449.1084 & $\mathrm{C}_{21} \mathrm{H}_{22} \mathrm{O}_{11}$ & $449 \rightarrow 303,285$ & isoastilbin & $\mathrm{Sa}, \mathrm{Sm}$ & {$[8,9]$} \\
\hline 68 & 9.87 & {$[\mathrm{M}-\mathrm{H}]$} & 451.1025 & 451.1029 & $\mathrm{C}_{24} \mathrm{H}_{20} \mathrm{O}_{9}$ & $451 \rightarrow 341,299$ & cinchonain $\mathrm{Ib}$ & $\mathrm{Sm}$ & \\
\hline 69 & 9.95 & {$[\mathrm{M}-\mathrm{H}]$} & 717.1442 & 717.1456 & $\mathrm{C}_{36} \mathrm{H}_{30} \mathrm{O}_{16}$ & $717 \rightarrow 519,475,321$ & caffeic acid tetramer isomer & A & \\
\hline 70 & 9.96 & {$[\mathrm{M}-\mathrm{H}]$} & 597.1605 & 597.1608 & $\mathrm{C}_{30} \mathrm{H}_{30} \mathrm{O}_{13}$ & $597 \rightarrow 451,341,217$ & glabraoside $\mathrm{C}$ & $\mathrm{Sa}$ & {$[8]$} \\
\hline 71 & 9.95 & {$[\mathrm{M}-\mathrm{H}]$} & 719.1599 & 719.1512 & $\mathrm{C}_{36} \mathrm{H}_{32} \mathrm{O}_{16}$ & $719 \rightarrow 539,359$ & dirosmarinic acid & $\mathrm{Sa}$ & \\
\hline 72 & 10.08 & {$[\mathrm{M}-\mathrm{H}]$} & 433.1131 & 433.1135 & $\mathrm{C}_{21} \mathrm{H}_{22} \mathrm{O}_{10}$ & $433 \rightarrow 343,313,271,179$ & $(2 R / 2 S)$-naringenin-6-C-glucopyranoside & $\mathrm{Sa}$ & [8] \\
\hline 73 & 10.17 & {$[\mathrm{M}-\mathrm{H}]$} & 433.1131 & 433.1135 & $\mathrm{C}_{21} \mathrm{H}_{22} \mathrm{O}_{10}$ & $433 \rightarrow 343,313,271,179$ & $(2 R / 2 S)$-naringenin-6-C-glucopyranoside & $\mathrm{Sa}$ & [8] \\
\hline 74 & 10.36 & {$[\mathrm{M}-\mathrm{H}]$} & 587.2327 & 587.2340 & $\mathrm{C}_{27} \mathrm{H}_{40} \mathrm{O}_{14}$ & $587 \rightarrow 451,341,217$ & sarcaglaboside D & $\mathrm{Sa}$ & {$[8]$} \\
\hline 75 & 10.61 & {$[\mathrm{M}-\mathrm{H}]$} & 515.1184 & 515.1190 & $\mathrm{C}_{25} \mathrm{H}_{24} \mathrm{O}_{12}$ & $515 \rightarrow 353$ & dicaffeoylquinic acid & $\mathrm{Sa}$ & \\
\hline 76 & 10.64 & {$[\mathrm{M}-\mathrm{H}]$} & 359.0768 & 359.0767 & $\mathrm{C}_{18} \mathrm{H}_{16} \mathrm{O}_{8}$ & $359 \rightarrow 197,161$ & rosmarinic acid & $\mathrm{Sa}, \mathrm{Sm}$ & {$[8,9]$} \\
\hline 77 & 10.73 & {$[\mathrm{M}-\mathrm{H}]$} & 279.1234 & 279.1232 & $\mathrm{C}_{15} \mathrm{H}_{20} \mathrm{O}_{5}$ & $279 \rightarrow 235,139$ & zedoalactone D & $\mathrm{Sa}$ & {$[8]$} \\
\hline 78 & 10.97 & {$[\mathrm{M}+\mathrm{COOH}]$} & 507.1497 & 461.1448 & $\mathrm{C}_{23} \mathrm{H}_{26} \mathrm{O}_{10}$ & $461 \rightarrow 417,295$ & lactiflorin & $\mathrm{P}$ & \\
\hline 79 & 11.03 & {$[\mathrm{M}-\mathrm{H}]$} & 433.1132 & 433.1135 & $\mathrm{C}_{21} \mathrm{H}_{22} \mathrm{O}_{10}$ & $433 \rightarrow 287,269$ & engeletin & $\mathrm{Sm}$ & {$[9]$} \\
\hline 80 & 11.41 & {$[\mathrm{M}-\mathrm{H}]$} & 423.1653 & 423.1655 & $\mathrm{C}_{21} \mathrm{H}_{28} \mathrm{O}_{9}$ & $423 \rightarrow 261,243$ & chloranoside A & $\mathrm{Sa}$ & {$[8]$} \\
\hline 81 & 11.80 & {$[\mathrm{M}-\mathrm{H}]$} & 549.1603 & 549.1608 & $\mathrm{C}_{26} \mathrm{H}_{30} \mathrm{O}_{13}$ & $549 \rightarrow 417,255$ & isoliquiritin apioside & G & {$[26]$} \\
\hline 82 & 11.94 & {$[\mathrm{M}-\mathrm{H}]$} & 433.1132 & 433.1135 & $\mathrm{C}_{21} \mathrm{H}_{22} \mathrm{O}_{10}$ & $433 \rightarrow 287,269$ & isoengeletin & $\mathrm{Sa}, \mathrm{Sm}$ & {$[8,9]$} \\
\hline 83 & 11.82 & {$[\mathrm{M}-\mathrm{H}]$} & 549.1607 & 549.1608 & $\mathrm{C}_{26} \mathrm{H}_{30} \mathrm{O}_{13}$ & $549 \rightarrow 417,255$ & liquiritin apioside & G & {$[26]$} \\
\hline 84 & 12.40 & {$[\mathrm{M}-\mathrm{H}]$} & 417.1183 & 417.1186 & $\mathrm{C}_{21} \mathrm{H}_{22} \mathrm{O}_{9}$ & $417 \rightarrow 255$ & isoliquiritin & G & {$[26]$} \\
\hline 85 & 12.97 & {$[\mathrm{M}-\mathrm{H}]$} & 599.1756 & 599.1765 & $\mathrm{C}_{30} \mathrm{H}_{32} \mathrm{O}_{13}$ & $599 \rightarrow 569$ & benzoyloxypaeoniflorin & $\mathrm{P}$ & {$[24]$} \\
\hline 86 & 12.98 & {$[\mathrm{M}-\mathrm{H}]$} & 451.1027 & 451.1029 & $\mathrm{C}_{24} \mathrm{H}_{20} \mathrm{O}_{9}$ & $451 \rightarrow 341,299$ & cinchonain Ic & $\mathrm{Sm}$ & [8] \\
\hline
\end{tabular}


Table 1. Cont.

\begin{tabular}{|c|c|c|c|c|c|c|c|c|c|}
\hline $\begin{array}{l}\text { Peak } \\
\text { No. }\end{array}$ & $\begin{array}{c}t \mathbf{R} \\
(\mathrm{min})\end{array}$ & $\begin{array}{c}\text { Selected } \\
\text { Ion }\end{array}$ & $\begin{array}{c}\text { Observed } \\
\text { Mass }(m / z)\end{array}$ & $\begin{array}{l}\text { Calculated } \\
\operatorname{Mass}(m / z)\end{array}$ & Formula & $\begin{array}{l}\text { MS/MS Patterns } \\
\text { Fragmentation }\end{array}$ & Identifieation & Source $^{\text {a }}$ & Reference \\
\hline 87 & 13.06 & {$[\mathrm{M}-\mathrm{H}]$} & 255.0658 & 255.0657 & $\mathrm{C}_{15} \mathrm{H}_{12} \mathrm{O}_{4}$ & $255 \rightarrow 135$ & liquiritigenin & $\mathrm{G}$ & {$[26]$} \\
\hline 88 & 13.14 & {$[\mathrm{M}-\mathrm{H}]$} & 451.1025 & 451.1029 & $\mathrm{C}_{24} \mathrm{H}_{20} \mathrm{O}_{9}$ & $451 \rightarrow 341,299$ & cinchonain Id & $\mathrm{Sm}$ & [8] \\
\hline 89 & 13.33 & {$[\mathrm{M}-\mathrm{H}]$} & 373.0918 & 373.0923 & $\mathrm{C}_{19} \mathrm{H}_{18} \mathrm{O}_{8}$ & $373 \rightarrow 211,161$ & methyl rosmarina & $\mathrm{Sa}, \mathrm{Sm}$ & [8] \\
\hline 90 & 13.52 & {$[\mathrm{M}-\mathrm{H}]$} & 823.4102 & 823.4116 & $\mathrm{C}_{42} \mathrm{H}_{64} \mathrm{O}_{16}$ & $823 \rightarrow 647,351$ & uralsaponin $\mathrm{C}$ & $\mathrm{G}$ & {$[27]$} \\
\hline 91 & 13.86 & {$[\mathrm{M}-\mathrm{H}]$} & 835.3742 & 835.3752 & $\mathrm{C}_{42} \mathrm{H}_{60} \mathrm{O}_{17}$ & $823 \rightarrow 661,351$ & uralsaponin D & G & [27] \\
\hline 92 & 13.52 & {$[\mathrm{M}-\mathrm{H}]$} & 999.4421 & 999.4433 & $\mathrm{C}_{48} \mathrm{H}_{72} \mathrm{O}_{22}$ & $999 \rightarrow 837,645,351$ & 24-hydroxyl-licorice-saponin A3 & G & [27] \\
\hline 93 & 13.64 & {$[\mathrm{M}-\mathrm{H}]$} & 895.3950 & 895.3964 & $\mathrm{C}_{44} \mathrm{H}_{64} \mathrm{O}_{19}$ & $895 \rightarrow 719,501,351$ & uralsaponin F & G & [27] \\
\hline 94 & 13.63 & {$[\mathrm{M}-\mathrm{H}]$} & 853.3845 & 853.3858 & $\mathrm{C}_{42} \mathrm{H}_{62} \mathrm{O}_{18}$ & $853 \rightarrow 809,791,677,351$ & 22-hydroxyl-licorice-saponin G2 & $\mathrm{G}$ & [27] \\
\hline 95 & 13.61 & {$[\mathrm{M}-\mathrm{H}]$} & 983.4470 & 983.4488 & $\mathrm{C}_{48} \mathrm{H}_{72} \mathrm{O}_{21}$ & $983 \rightarrow 821,645,351$ & licorice saponin A3 & $\mathrm{G}$ & {$[27]$} \\
\hline 96 & 13.68 & {$[\mathrm{M}-\mathrm{H}]$} & 1025.4579 & 1025.4593 & $\mathrm{C}_{50} \mathrm{H}_{74} \mathrm{O}_{22}$ & $1025 \rightarrow 993,833,497$ & 22-acetoxyl-rhaoglycyrrhizin & $\mathrm{G}$ & {$[27]$} \\
\hline 97 & 13.70 & {$[\mathrm{M}-\mathrm{H}]$} & 849.3538 & 849.3545 & $\mathrm{C}_{42} \mathrm{H}_{58} \mathrm{O}_{18}$ & $849 \rightarrow 673,479$ & uralsaponin $\mathrm{E}$ & $\mathrm{G}$ & {$[27]$} \\
\hline 98 & 13.76 & {$[\mathrm{M}-\mathrm{H}]$} & 879.3996 & 879.4014 & $\mathrm{C}_{44} \mathrm{H}_{64} \mathrm{O}_{18}$ & $879 \rightarrow 861,643,351$ & 22-acetoxyl-glycyrrhizin & G & [27] \\
\hline 99 & 13.77 & {$[\mathrm{M}-\mathrm{H}]$} & 837.3891 & 837.3909 & $\mathrm{C}_{42} \mathrm{H}_{62} \mathrm{O}_{17}$ & $837 \rightarrow 819,661,351$ & 24-hydroxyl-glycyrrhizin & G & [27] \\
\hline 100 & 13.78 & {$[\mathrm{M}-\mathrm{H}]$} & 271.0607 & 271.0606 & $\mathrm{C}_{15} \mathrm{H}_{12} \mathrm{O}_{5}$ & $271 \rightarrow 254,177$ & naringenin & $\mathrm{Sm}$ & {$[27]$} \\
\hline 101 & 13.86 & {$[\mathrm{M}-\mathrm{H}]$} & 835.3742 & 835.3752 & $\mathrm{C}_{42} \mathrm{H}_{60} \mathrm{O}_{17}$ & $823 \rightarrow 661,351$ & 24-hydroxyl-licorice-saponin E2 & $\mathrm{G}$ & {$[27]$} \\
\hline 102 & 14.00 & {$[\mathrm{M}-\mathrm{H}]$} & 837.3891 & 837.3909 & $\mathrm{C}_{42} \mathrm{H}_{62} \mathrm{O}_{17}$ & $837 \rightarrow 819,775,661,351$ & licorice saponin G2 & $\mathrm{G}$ & {$[27]$} \\
\hline 103 & 14.00 & {$[\mathrm{M}-\mathrm{H}]$} & 967.4523 & 967.4539 & $\mathrm{C}_{48} \mathrm{H}_{72} \mathrm{O}_{20}$ & $967 \rightarrow 805,497,407,321$ & rhaoglycyrrhizin & $\mathrm{G}$ & {$[27]$} \\
\hline 104 & 14.01 & {$[\mathrm{M}-\mathrm{H}]$} & 819.3787 & 819.3803 & $\mathrm{C}_{42} \mathrm{H}_{60} \mathrm{O}_{16}$ & $819 \rightarrow 777,643,351$ & licorice saponin E2 & G & [27] \\
\hline 105 & 14.06 & {$[\mathrm{M}-\mathrm{H}]$} & 863.4049 & 863.4065 & $\mathrm{C}_{44} \mathrm{H}_{64} \mathrm{O}_{17}$ & $\begin{array}{c}863 \rightarrow 819,729,687,351, \\
289\end{array}$ & 22-acetoxyl-glycyrrhaldehyde & $\mathrm{G}$ & {$[27]$} \\
\hline 106 & 14.13 & {$[\mathrm{M}-\mathrm{H}]$} & 255.0660 & 255.0657 & $\mathrm{C}_{15} \mathrm{H}_{12} \mathrm{O}_{4}$ & $255 \rightarrow 135$ & isoliquiritigenin & $\mathrm{G}$ & {$[27]$} \\
\hline 107 & 14.14 & {$[\mathrm{M}-\mathrm{H}]$} & 821.3945 & 821.3945 & $\mathrm{C}_{42} \mathrm{H}_{62} \mathrm{O}_{16}$ & $821 \rightarrow 803,759,645,351$ & glycyrrhizin & $\mathrm{G}$ & [27] \\
\hline 108 & 14.33 & {$[\mathrm{M}-\mathrm{H}]$} & 821.3945 & 821.3960 & $\mathrm{C}_{42} \mathrm{H}_{62} \mathrm{O}_{16}$ & $821 \rightarrow 803,759,645,351$ & 18a-glycyrrhizin & $\mathrm{G}$ & [27] \\
\hline
\end{tabular}

Note: a: A, Arnebiae radix; C, Curcumae rhizome; G, Glycyrrhizae radix et rhizome; M, Mume fructus; P, Paeoniae radix rubra; Sa, Sarcandrae Herba; Sm, Smilacis glabrae rhizome. 


\subsection{Method Validation of the Quantitative Analysis}

The calibration curves, linear ranges, LOQ, and repeatability of the 14 analytes were established using the developed UHPLC-MS method (Table 2). Reasonable correlation coefficient values $\left(r^{2}>0.9981\right)$ indicated good correlations between the investigated concentrations of the standards and their peak areas within the ranges tested. The ranges of LOQ for all the analytes were from 0.013 to $0.065 \mu \mathrm{g} / \mathrm{mL}$.

Table 2. Calibration curves, linear range, LOQ and repeatability for fourteen compounds analyzed with the UHPLC-MS system.

\begin{tabular}{cccccc}
\hline Analyte & $\begin{array}{c}\text { Linear Range } \\
(\boldsymbol{\mu g} / \mathbf{m L})\end{array}$ & Calibration Curve $(\boldsymbol{n}=\mathbf{7})$ & $\boldsymbol{r}^{\mathbf{2}}$ & $\begin{array}{c}\text { LOQ } \\
(\boldsymbol{\mu g} / \mathbf{m L})\end{array}$ & $\begin{array}{c}\text { Repeatability } \\
\text { RSD (\%) }\end{array}$ \\
\hline 3-O-Caffeoylquinic acid (16) & $0.16-6.37$ & $y=301,311 x-46,304$ & 0.9984 & 0.064 & 2.3 \\
5-O-Caffeoylquinic acid (22) & $0.23-9.24$ & $y=341,667 x-39,368$ & 0.9982 & 0.046 & 3.2 \\
4-O-Caffeoylquinic acid (24) & $0.41-16.34$ & $y=267,929 x-80,795$ & 0.9990 & 0.065 & 3.8 \\
5-O-Caffeoylskimic acid (39) & $0.57-22.92$ & $y=396,547 x+152,855$ & 0.9985 & 0.057 & 3.5 \\
Paeoniflorin (40) & $2.54-101.62$ & $y=207,558 x+1,315,316$ & 0.9988 & 0.040 & 1.7 \\
Liquiritin (51) & $0.46-18.24$ & $y=616,184 x+109,090$ & 0.9987 & 0.036 & 1.8 \\
Neoastilbin (56) & $0.47-18.84$ & $y=572,723 x+139,006$ & 0.9983 & 0.016 & 1.7 \\
Astilbin (60) & $0.55-22.16$ & $y=500,903 x+165,817$ & 0.9990 & 0.055 & 1.5 \\
Neoisoastilbin (64) & $0.39-15.77$ & $y=339,480 x+93,661$ & 0.9986 & 0.067 & 2.0 \\
Isoastilbin (67) & $0.32-12.84$ & $y=531,497 x-76,292$ & 0.9984 & 0.018 & 1.5 \\
Engeletin (79) & $0.31-12.50$ & $y=743,986 x-120,215$ & 0.9983 & 0.013 & 2.1 \\
Isoengeletin (82) & $0.67-26.66$ & $y=355,937 x+110,394$ & 0.9987 & 0.027 & 3.8 \\
Liquiritigenin (87) & $0.07-2.96$ & $y=426,840 x-24,980$ & 0.9981 & 0.030 & 2.0 \\
glycyrrhizic acid (107) & $0.99-39.56$ & $y=422,502 x+468,934$ & 0.9986 & 0.040 & 1.6 \\
\hline
\end{tabular}

Table 3. Intra-day and inter-day precisions and recoveries for fourteen compounds analyzed with the UHPLC-MS system.

\begin{tabular}{cccccccc}
\hline & $\begin{array}{c}\text { Intra-Day } \\
\text { Analyte }\end{array}$ & $\begin{array}{c}\text { Inter-Day } \\
(\mathbf{R S D}, \mathbf{\%})\end{array}$ & \multicolumn{5}{c}{ Recoveries $(\boldsymbol{n}=\mathbf{6})$} \\
\cline { 5 - 8 } & $(\mathbf{n = 6 )}$ & $(\boldsymbol{n}=\mathbf{3})$ & $\mathbf{( \mu g )}$ & $\begin{array}{c}\text { Spiked } \\
(\boldsymbol{\mu g})\end{array}$ & $\begin{array}{c}\text { Detected } \\
(\boldsymbol{\mu g})\end{array}$ & $\begin{array}{c}\text { Recoveries } \\
(\mathbf{\%})\end{array}$ & $\begin{array}{c}\text { RSD } \\
(\mathbf{\%})\end{array}$ \\
\hline 3-O-Caffeoylquinic acid (16) & 2.9 & 2.8 & 1.71 & 1.75 & 3.23 & 92.6 & 4.8 \\
5-O-Caffeoylquinic acid (22) & 1.3 & 4.4 & 1.38 & 1.27 & 2.87 & 108.4 & 3.8 \\
4-O-Caffeoylquinic acid (24) & 1.3 & 3.4 & 2.07 & 2.25 & 4.51 & 104.1 & 3.4 \\
5-O-Caffeoylskimic acid (39) & 0.6 & 3.7 & 2.99 & 3.15 & 5.54 & 90.2 & 1.4 \\
Paeoniflorin (40) & 3.2 & 2.7 & 27.34 & 27.95 & 57.69 & 104.5 & 3.8 \\
Liquiritin (51) & 2.9 & 2.5 & 4.95 & 5.02 & 10.05 & 101.3 & 2.5 \\
Neoastilbin (56) & 1.9 & 3.1 & 5.25 & 5.18 & 11.38 & 109.5 & 2.3 \\
Astilbin (60) & 3.4 & 3.0 & 6.02 & 6.10 & 11.86 & 97.8 & 3.7 \\
Neoisoastilbin (64) & 3.0 & 3.2 & 4.24 & 4.34 & 8.21 & 95.6 & 3.9 \\
Isoastilbin (67) & 3.2 & 3.1 & 3.60 & 3.53 & 7.49 & 105.1 & 3.2 \\
Engeletin (79) & 2.5 & 2.8 & 3.31 & 3.44 & 6.99 & 104.1 & 4.1 \\
Isoengeletin (82) & 3.8 & 2.3 & 2.51 & 2.62 & 4.83 & 94.4 & 3.0 \\
Liquiritigenin (87) & 2.3 & 2.6 & 0.82 & 0.81 & 1.52 & 93.2 & 2.4 \\
glycyrrhizic acid (107) & 0.8 & 1.5 & 10.75 & 10.88 & 22.83 & 106.0 & 2.1 \\
\hline
\end{tabular}


The repeatability presented as $\operatorname{RSD}(n=5)$ was between $1.5 \%$ and $3.8 \%$ for the 14 compounds. The overall intra- and inter-day variations (RSD) of the 14 analytes were in the range from $0.6 \%$ to $3.8 \%$, and 1.5 to $4.4 \%$ (Table 3), respectively. The developed method had good accuracy with the recoveries were between $86.3 \%$ and $109.5 \%$ (Table 3). Therefore, the results demonstrated that the UHPLC-ESI-MS method was sensitive, precise, and accurate enough for quantitative evaluation of multi-compounds in PSORI-CM01 preparations.

\subsection{Quantitative Determination of PSORI-CM01 Preparations}

A total of eight different batches of PSORI-CM01 preparations were tested using the developed LC-ESI-MS method. The contents $(n=3)$ of 14 investigated compounds are summarized in Table 4. It was recognized that 3-O-caffeoylquinic acid (16), 5- $O$-caffeoylquinic acid (22), 4-O-caffeoylquinic acid (24), 5-O-caffeoylskimic acid (39), paeoniflorin (40), liquiritin (51), neoastilbin (56), astilbin (60), neoisoastilbin (64), isoastilbin (67), and glycyrrhizic acid (107) were the dominant compounds in all examined samples. However, the contents of each compound or the total content of certain type of constituents varied in different PSORI-CM01 preparation.

Table 4. The contents of the 14 compounds in different batches of PSORI-CM01 prepations $(n=3)$.

\begin{tabular}{ccccccccc}
\hline Analyte $^{\text {a }}$ & KLJ-1 & KLJ-2 & PJ-3 & PJ-4 & PJ-5 & TJ-6 & TJ-7 & TJ-8 \\
\hline 3-O-Caffeoylquinic acid (16) & 520.94 & 531.48 & 391.56 & 132.01 & 429.07 & 122.89 & 30.68 & 121.18 \\
5-O-Caffeoylquinic acid (22) & 514.55 & 503.94 & 391.56 & 146.51 & 488.10 & 123.45 & 27.30 & 183.64 \\
4-O-Caffeoylquinic acid (24) & 601.74 & 545.00 & 558.82 & 211.85 & 683.57 & 129.75 & 40.53 & 160.64 \\
5-O-Caffeoylskimic acid (39) & 540.55 & 520.10 & 789.39 & 324.27 & 397.06 & 333.60 & 153.44 & 429.46 \\
Paeoniflorin (40) & 5855.02 & 6030.52 & 7218.12 & 5177.23 & 5368.14 & 2326.21 & 445.51 & 3511.46 \\
Liquiritin (51) & 1654.18 & 1650.73 & 892.15 & 360.64 & 1115.65 & 145.56 & 71.55 & 248.20 \\
Neoastilbin (56) & 2545.86 & 2767.29 & 2442.62 & 423.15 & 1680.52 & 369.88 & 124.41 & 588.89 \\
Astilbin (60) & 3819.23 & 4061.92 & 3743.95 & 844.53 & 2575.83 & 661.96 & 174.14 & 879.05 \\
Neoisoastilbin (64) & 2459.06 & 2359.70 & 1872.94 & 736.75 & 2211.36 & 605.51 & 154.36 & 802.79 \\
Isoastilbin (67) & 879.60 & 916.61 & 650.53 & 260.87 & 902.06 & 244.03 & 74.96 & 418.97 \\
Engeletin (79) & 678.32 & 740.00 & 503.10 & 196.27 & 621.69 & 165.13 & 84.25 & 274.81 \\
Isoengeletin (82) & 543.19 & 1089.55 & 348.12 & 132.29 & 331.67 & 119.27 & 16.30 & 116.39 \\
Liquiritigenin (87) & 224.07 & 246.07 & 477.28 & 62.14 & 202.73 & 75.50 & 24.55 & 130.18 \\
glycyrrhizic acid (107) & 2225.13 & 2359.89 & 2610.50 & 933.51 & 2770.51 & 306.01 & 68.44 & 257.72 \\
\hline
\end{tabular}

Notes: ${ }^{a}$ The content unit of granule (KLJ-1, KLJ-2) and pills (PJ-3, PJ-4, PJ-5) was expressed as $\mu \mathrm{g} / \mathrm{g}$; The content unit of decoction was $\mathrm{ug} / \mathrm{mL}$.

In order to evaluate the variations in detail, hierarchical cluster analysis was performed based on the contents of 14 analytes of the eight investigated batches. Between-groups linkages method was applied, and Squared Euclidean distance was selected as measurement. Figure 2 shows the results on the investigated batches of PSORI-CM01 preparations, which were divided into two main clusters. The results suggested that the contents of 14 analytes were relatively more stable and higher in granule preparations (the batches of $\mathrm{K} 1$ and $\mathrm{K} 2$ ) but varied in tablet and decoction preparations. This may be related to the origin of raw medicinal plants origin and extraction technology and so on. As mentioned 
above, 3-O-caffeoylquinic acid (16), paeoniflorin (40), liquiritin (51), astilbin (60), engeletin (79), liquiritigenin (87) and glycyrrhizic acid (107) may be the main active components of PSORI-CM01, The content of the six ingredients should be stressed on in establishing quality control methods for PSORI-CM01 preparations.

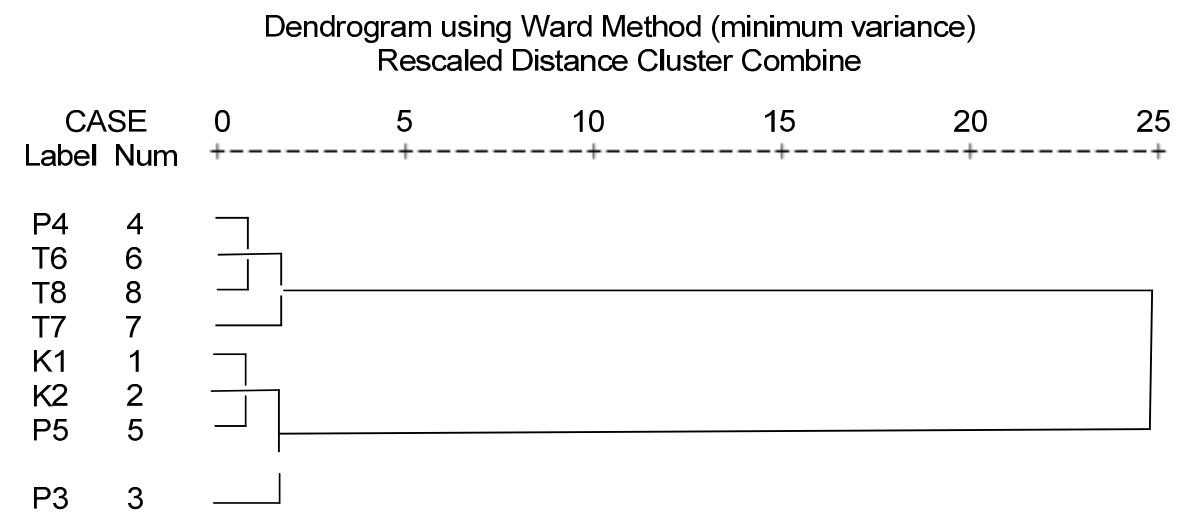

Figure 2. Dendrogram of hierarchical cluster analysis for the eight investigated batches of PSORI-CM01 preparations.

\section{Experimental Section}

\subsection{Chemicals and Materials}

Methanol and acetonitrile (HPLC grade) were purchased from Fisher Scientific (Fair Lawn, NJ, USA); Formic acid (HPLC grade) was purchased from the Sigma-Aldrich (Seelze, Germany); Ultra-pure water was prepared using a Millipore Milli-Q purification system (Bedford, MA, USA).

5-O-caffeoylskimic acid (39), paeoniflorin (40), neoastilbin (56), astilbin (60), neoisoastilbin (64), isoastilbin (67), engeletin (79) and isoengeletin (82) were isolated in our lab and identified by the authors. 3-O-caffeoylquinic acid (16), 5-O-caffeoylquinic acid (22), 4- $O$-caffeoylquinic acid (24), liquiritin (51), liquiritigenin (87), and glycyrrhizic acid (107) were purchase from the National Institutes for Food and Drug Control (Beijing, China). All of the purities were above 98\% by HPLC analysis. Eight batches of PSORI-CM01 preparations were supplied by Guangdong Provincial Hospital of Chinese Medicine, and voucher samples were deposited in the laboratory of Materia Medica Preparation, Guangdong Province Academy of Chinese Medicine Science.

\subsection{Standard Solutions and Sample Preparation}

Stock solutions of the 14 reference substances were prepared in concentrations ranging from 0.420 to $5.083 \mathrm{mg} / \mathrm{mL}$ in $60 \%$ methanol and stored at $4{ }^{\circ} \mathrm{C}$ until use. A standard working solution of the mixtures was obtained by diluting stock solutions to desired concentrations. Aliquots of this solution were further diluted with initial mobile phase to a series of concentrations for quantification.

PSORI-CM01 preparations (granules and tablets) were pulverized into a fine powder. The powder $(0.5 \mathrm{~g})$ was accurately weighed, immersed in $60 \% \mathrm{MeOH}(\mathrm{v} / \mathrm{v}, 10 \mathrm{~mL})$ for $1 \mathrm{~h}$ at room temperature, then extracted in an ultrasonic water bath for $30 \mathrm{~min}$. After recording the weight, the extract was filtered through filter paper. Aliquots of $500 \mu \mathrm{L}$ of filtrate was transferred into a $5 \mathrm{~mL}$ volumetric flask 
which was made up to its volume with initial mobile phase. PSORI-CM01 decoction was diluted 10 times in $60 \% \mathrm{MeOH}(\mathrm{v} / \mathrm{v})$ for quantitative analysis. All of the samples were filtered through a $0.22 \mu \mathrm{m}$ syringe filter before use, and $10 \mu \mathrm{L}$ was injected into the LC instrument for LC-MS analysis.

\subsection{UHPLC-ESI-MS/MS System}

Chromatographic separation was performed on an Accela ${ }^{\mathrm{TM}}$ ultra high pressure liquid chromatography (UHPLC) system (Thermo Fisher Scientific, San Jose, CA, USA) comprising a UHPLC pump, a PDA detector, scanning from 200 to $400 \mathrm{~nm}$, and an autosampler settled to $30^{\circ} \mathrm{C}$. The LC conditions were as follows: column: Thermo Scientific Syncronis C18 $(50 \mathrm{~mm} \times 2.1 \mathrm{~mm}, 1.7 \mu \mathrm{m})$; mobile phase: acetonitrile (A) and water containing $0.1 \%(\mathrm{v} / \mathrm{v})$ formic acid (B); gradient: $0 \mathrm{~min}, 5: 95 ; 10 \mathrm{~min}, 27: 83$; $15 \mathrm{~min}, 50: 50 ; 18-20 \mathrm{~min}, 100: 0(\mathrm{~A}: \mathrm{B}, \mathrm{v} / \mathrm{v})$; flow rate: $0.4 \mathrm{~mL} / \mathrm{min}$; injection volume: $10 \mu \mathrm{L}$.

The above UHPLC system was connected with a LTQ/Orbitrap mass spectrometry system (Thermo-Fisher Scientific, Bremen, Germany) via an ESI interface. High purity nitrogen $\left(\mathrm{N}_{2}\right)$ was used as the sheath gas and helium $(\mathrm{He})$ as the auxiliary gas with a flow rate of 40 and 10 arbitrary units, respectively.

\subsection{Qualitative Characteristic of Chemical Constituents}

Identification of chemical constituents in PSORI-CM01 preparations was performed by LC-ESI-MS $^{\mathrm{n}}$ analysis. The ESI-MS spectra of samples and reference compounds were acquired in negative ionization mode. The parameters were as follows: capillary temperature at $320{ }^{\circ} \mathrm{C}$, capillary voltage at $-28 \mathrm{~V}$, ion spray voltage at $-4.0 \mathrm{kV}$, and tube lens voltage at $-90 \mathrm{~V}$. For full scan MS analysis, the spectra were recorded in the range of $m / z 100-1500$ with a resolution of 30,000. Data-dependant acquisition was applied and the most intense ions detected in each MS scan were selected for $\mathrm{MS}^{\mathrm{n}}$ data records with a resolution of 15,000 . The activation time was $30 \mathrm{~ms}$ and the collision energy was adjusted to 35\%. Data were processed by Xcalibur software (Thermo-Fisher Scientific, Bremen, Germany). An external calibration for mass accuracy was carried out the day before the analysis according to the manufacturer's guidelines.

\subsection{Validation of the Quantitative Analysis}

The stock solution containing 14 reference compounds was prepared and diluted to seven-point calibration levels for the construction of calibration curves. Each concentration of the mixed standard solution was injected in triplicate. Calibration curves were established by plotting the peak area versus concentration of each analyte. Intra- and inter-day variations were utilized to assess the precision of the method. The intra-day variation was determined by analyzing six replicates within 1 day and the inter-day variation was examined in three consecutive days. Recovery was used to evaluate the accuracy of the method. Recovery test was carried out to investigate accuracy of this method by adding certain amounts of the 14 standard solutions to $0.25 \mathrm{~g}$ powder of sample in sextuplicate. Samples were processed as described in Section 2.2. To confirm the repeatability, five replicates of the same sample were extracted and analyzed. Variations were expressed by relative standard deviation (RSD) in all three tests above. 


\section{Conclusions}

An efficient and sensitive method employing ultra-high liquid chromatography coupled with linear trap quadrupole and high resolution mass analyzer-orbitrap (UHPLC-LTQ/Orbitrap) was developed for the qualitative and quantitative analysis of chemical constituents of PSORI-CM01 preparations. One hundred and eight compounds, including organic acids, phenolic acids, flavonoids, and terpenoids, were characterized on the basis of retention behaviors, abundant MS and $\mathrm{MS}^{\mathrm{n}}$ data, or by comparing with reference substances and literatures. All compounds identified were found to be existed in individual traditional Chinese medicines of PSORI-CM01 preparation. However, the characteristic constituents from Rhizoma Curcumae were not detected. Further investigation focused on those lipophilic constituents in PSORI-CM01 preparation is required.

An optimized LC-ESI-MS method was then established for assay of the 14 marker compounds in PSORI-CM01 preparations. The validation of the method, represent a good accuracy, sensitivity and repeatability. The quantification results indicated an obvious difference of marker compounds contents among various samples. This is the first report on the comprehensive determination of chemical constituents in PSORI-CM01 preparations by LC-ESI-MS ${ }^{n}$. The results would provide the chemical support for the further pharmacokinetic studies and for the improvement of quality control of PSORI-CM01 preparations. The study also suggested that LC-LTQ/Orbitrap-MS would be a powerful and reliable analytical tool for the characterization of chemical profile in complex chemical system, such as TCM preparations.

\section{Acknowledgments}

This research was financially supported by Guangdong Natural Science Fund (S2013030011515), Guangdong Financial Industry Technology Research Development Fund [2011(285)05], Guangdong Science and Technology Department-Guangdong Province Academy of Chinese Medicine Science Joint Special Fund (2011B032200009) and Guangdong Provincial Hospital of Chinese Medicine Special Fund (YK2013B1N11).

\section{Author Contributions}

C.-J. Lu designed the experiments and provided critical advice on operation of the analytical equipment. S.-D. Chen was responsible for performing most of the experiment and analysis, and preparing the draft of the manuscript. R.-Z. Zhao helped revising the manuscript.

\section{Conflicts of Interest}

The authors declare no conflict of interest.

\section{References}

1. Yao, X.S.; Ye, W.C.; Kurihara, H. Reveal the scientific connotation of TCM for promoting its modernization and innovative drug research process. Prog. Chem. 2009, 21, 2-13. 
2. Qin, K.M.; Cai, H.; Zhang, L.; Shi, Y.; Li, P.; Cai, B.C. Chemical constituents and effective substances of traditional Chinese eedicinal formula. Prog. Chem. 2010, 22, 2436-2449.

3. Nagler, E.V.; Webster, A.C.; Vanholder, R.; Zoccali, C. Antidepressants for depression in stage 3-5 chronic kidney disease: A systematic review of pharmacokinetics, efficacy and safety with recommendations by European Renal Best Practice (ERBP). Nephrol. Dial. Transplant. 2012, 27, 3736-3745.

4. Lu, C.J.; Xiang, Y.; Xie, X.L.; Xuan, M.L.; He, Z.H. A randomized controlled single-blind clinical trial on 84 outpatients with psoriasis vulgaris by auricular therapy combined with optimized Yinxieling formula. Chin. J. Inter. Med. 2012, 3, 186-191.

5. Wang, L.; Huang, Y.Q. Clinical observation of Yinxieling tablets on treating psoriasis vulgaris. Guangzhou Zhongyiyao Daxue Xuebao 2009, 26, 520-521.

6. Zhong, J.B.; Yin, X.; Lu, C.J.; Xuan, G.W. Experience introduction of Xuan Guo Wei professor in treating psoriasis. J. New Chin. Med. 2004, 9, 11-12.

7. Liu E.H.; Qi, L.W.; Li, B.; Peng, Y.B.; Li, P.; Li, C.Y.; Cao, J. High-speed separation and characterization of major constituents in Radix Paeoniae Rubra by fast highperformance liquid chromatography coupled with diode-array detection and time-of-flight mass spectrometry. Rapid Commun. Mass Spectrom. 2009, 23, 119-130.

8. Li, X.; Zhang, Y.F.; Zeng, X.; Yang, L.; Deng, Y.H. Chemical profiling of bioactive constituents in Sarcandra glabra and its preparations using ultra-high-pressure liquid chromatography coupled with LTQ Orbitrap mass spectrometry. Rapid Commun. Mass Spectrom. 2011, 25, 2439-2447.

9. Chen, S.D.; Lu, C.J.; Zhao, R.Z. Qualitative and quantitative analysis of Rhizoma Smilacis glabrae by ultra high performance liquid chromatography coupled with LTQ Orbitrap ${ }^{\mathrm{XL}}$ hybrid mass spectrometry. Molecules 2014, 19, 10427-10439.

10. Bai, G.; Jin, X.J. Chemical constituents of Lithospermum erythrorhizon. Chem. Res. Chin. Univ. 1994, 10, 263-265.

11. Kawata, J.; Kameda, M.; Miyazawa, M. Cyclooxygenase-2 inhibitory effects and composition of the volatile oil from the dried roots of Lithospermum erythrorhizon. J. Nat. Med. 2008, 62, 239-243.

12. Chen, Z.G.; En, B.T.; Zhang, Z.Q. Simultaneous determination of eight organic acids in Fructus Mume by RP-HPLC. China J. Chin. Mater. Med. 2006, 31, 1783-1786.

13. Zhang, Q.Y.; Ye, M. Chemical analysis of the Chinese herbal medicine Gan-Cao (licorice). J. Chromatogr. A 2009, 1216, 1954-1969.

14. Phan, M.G.; Tran, T.T.N.; Phan, T.S.; Matsunami, K.; Otsuka, H. Guaianolides from Curcuma kwangsiensis. Phytochem. Lett. 2014, 9, 137-140.

15. Li, J.; Zhao, F.; Li, M.Z.; Chen, L.X.; Qiu, F. Diarylheptanoids from the Rhizomes of Curcuma kwangsiensis. J. Nat. Prod. 2010, 73, 1667-1671.

16. Ye, M; Li, Y; Yan, Y.N.; Liu, H.W.; Ji, X.H. Determination of flavonoids in Semen Cuscutae by RP-HPLC. J. Pharm. Biomed. Anal. 2002, 28, 621-628.

17. Su, Z.H.; Zou, G.A.; Preiss, A.; Zhang, H.W.; Zou, Z.M. Online identification of antioxidant constituents of traditional Chinese medicine formular Chaihu-Shu-Gan-San by LC-LTQ-Orbitrap mass spectrometry and microplate spectrophotometer. J. Pharm. Biomed. Anal. 2010, 53, 454-461.

18. Wu, W.H.; Kang, Z.; Ouyang, D.S.; Zhou, H.H. Progresses in the pharmacology of chlorogenic acid. Nat. Prod. Res. Dev. 2006, 18, 691-694. 
19. Zheng, Y.Q.; Wei, W.; Zhu, L.; Liu, J.X. Effects and mechanisms of Paeoniflorin, a bioactive glucoside from paeony root, on adjuvant arthritis in rats. Inflamm. Res. 2007, 56, 182-188.

20. Goto, H.; Shimada, Y.; Akechi, Y.; Kohta, K.; Hattori, M.; Terasawa, K. Endothelium-dependent vasodilator effect of extract prepared from the roots of Paeonia lactiflora on isolated rat aorta. Planta Med. 1996, 62, 436-439.

21. Huang, H.Q.; Cheng, Z.H.; Shi, H.M.; Xin, W.B.; Wang, T.T.Y.; Yu, L.L. Isolation and characterization of two flavonoids, engeletin and astilbin, from the leaves of Engelhardia roxburghiana and their potential anti-inflammatory properties. J. Agric. Food. Chem. 2011, 59, 4562-4569.

22. Jia, G.H.; Jia, S.S. Pharmacological activities of flavonoids from licorice. Chin. Pharm. J. 1998, $33,513-516$.

23. Wang, X.F.; Shan F.P. Advance in glycyrrhizin's anti-inflammation and antitumor mechanism. J. Microbiol. 2013, 33, 88-92.

24. Zhang, H.J.; Shen, P.; Cheng, Y.Y. Identification and determination of the major constituents in traditional Chinese medicine Si-Wu-Tang by HPLC coupled with DAD and ESI-MS. J. Pharm. Biomed. Anal. 2004, 34, 705-713.

25. Wang, Z.J.; Woa, S.K.; Wang, L.; Lau, C.B.S.; Lee, V.H.L.; Chow, M.S.S.; Zuo, Z. Simultaneous quantification of active components in the herbs and products of Si-Wu-Tang by high performance liquid chromatography-mass spectrometry. J. Pharm. Biomed. Anal. 2009, 50, 232-244.

26. Zhou, Y.; Wang, M.K.; Liao, X.; Zhu, X.M.; Peng, S.L.; Ding, L.S. Rapid identification of compounds in Glycyrrhiza Uralensis by liquid chromatography/tandem mass spectrometry. Chin. J. Anal. Chem. 2004, 32, 174-178.

27. Zheng, Y.F.; Qi, L.W.; Zhou, J.L.; Li, P. Structural characterization and identification of oleananetype triterpene saponins in Glycyrrhiza uralensis Fischer by rapid-resolution liquid chromatography coupled with time-of-flight mass spectrometry. Rapid Commun. Mass Spectrom. 2010, 24, 3261-3270.

Sample Availability: Samples of the compounds 16, 22, 24, 39, 40, 51, 56, 60, 64, 67, 79, 82, 87 and $\mathbf{1 0 7}$ are available from the authors.

(C) 2015 by the authors; licensee MDPI, Basel, Switzerland. This article is an open access article distributed under the terms and conditions of the Creative Commons Attribution license (http://creativecommons.org/licenses/by/4.0/). 